\title{
Telephonic Consultation: Modern Era of some Unsolved Questions
}

\author{
Patel $\mathbf{U}^{1}$, Gedam DS ${ }^{2}$, Patel NP ${ }^{3}$, Ratre BK \\ Dr. Umesh Patel, Associate Professor of Pediatrics, Dr D Sharad Gedam, Professor of Pediatrics, Dr. Narmada Prasad Patel, \\ Assistant Professor of Medicine), Dr Bhupendra Kumar Ratre, Associate Professor of Medicine. All are affiliated with L N \\ Medical College, Bhopal MP, India
}

Address for Correspondence: Dr Umesh Patel, Email: drumeshpatel@gmail.com

\begin{abstract}
Improving access to healthcare is a major challenge for patients, doctors and community. Globally many traditional services which used to be delivered face to face (e.g. banking and shopping) are delivered now telephonically and electronically. Currently, telephonic consultation appears to be used for many different purposes despite scanty information and evidence on quality of method, pitfalls and limitations. However, despite the widespread use of telephones in daily life and the range of possible benefits that telephones may offer in medical services, their role in clinical practice remains highly controversial. Medical community find it difficult and less rewarding because of many factors and limitations. There is a need for focused research, proper training of medical professionals, specific guidelines on medico legal aspect and issue of professional fee need to be addressed for better utilization of seemingly useful and cost-effective method of telephonic consultation in clinical practice.
\end{abstract}

Key words: Telephonic consultation, Technology, Healthcare, Consultation

\section{Introduction}

We live in the era of technology. Technology dominates every movement of our day, right from the movement we get up in morning. There has been an explosive growth in the use of the telephone in all areas of life, from telephone banking, insurance, shopping and not least Medicine. In many countries general practice is also shifted from home visits to telephone advice.

Providing telephone advice is not new in health care, there are reports as early as 1897 [1]. Since its invention in 1876 the telephone has been used as a tool for delivering health care: Alexander Graham Bell's first recorded telephone call was for medical help after he spilt sulphuric acid on himself [2]. Consultations on the telephone are increasing in primary care. With increasing reliance on telephone consultations, healthcare delivery using this method is becoming more widely acceptable to members of the public and healthcare professionals.

There is evidence that many doctors find providing telephone advice stressful, often because of mismatch between the caller's expectations and doctors perceptions of their needs [3]. Little is known about the content of telephone consultations used for different purposes (e.g. acute triage, follow-up consultations, and chronic disease management) or the quality of the advice given by telephone in comparison with face-to-face consulting [4]. However like any other communication skill, it requires training and up-skilling. Provided that healthcare staff has been trained in dealing with telephone consultations, it can improve patient satisfaction and quality of care offered.

Telephone consultations require specific skills to compensate for the lack of visual clues that become apparent the movement the patient enters in the consultation room and to manage patient expectations for a home/clinic visit. A full clinical assessment is therefore not possible, but if the limitations of the telephone consultation are recognized, and a careful history is taken and documented, patients can be manage in a reasonable, appropriate and safe way.

It must be acknowledged that telephone consultations lack the nuances and richness of the face-to-face consultation. It is important to remember that you must put yourself in a position to justify the diagnosis and management plan you make in the context of a telephone consultation, and if there is any doubt then a face-to-face consultation should be arranged.

\section{Benefits}

\section{To the patient [5]}

- Speed of access and more convenient

- Increases access for those:

- Without their own means of transport.

- With a physical or psychological disability and restricted mobility 
- For those in work or their dependants.

- Time efficient for all parties

- In chronic disease management, telephone consultations have better compliance than follow-up visit.

- Easier access to self-care advice and patient education.[6]

- Easy access to advice as circumstances change

- When geographical distances separating the patient, this may be the only realistic means of seeking help.

To the doctor

- Improving efficiency by moving the information instead of the patient.

- Can be used to provide a triage system for same day, when appointment demand is high.[7]

- Can reduce workload - about 50\% telephone consults result in telephone advice alone. [7]
To both

- Reduced visits may mean less use of cars and other transport means and a potential environmental saving in terms of traffic congestion and carbon footprint.

\section{Drawbacks of telephonic consultation}

- Lack of non-verbal clues

- No direct observations and direct examination. It is very much dependent on the individual patient's perception of the problem. If someone downplays the symptoms, it can lead to potentially life-threatening mistakes.

- No smells or active listening

- No diagnostic tests

- Usually third-party consultations

- Hearing difficulties, accents difficulties, Cultural and language obstacles. It is not a very good tool for those who do not speak the native language,

- Technical difficultie

Table 1: Common errors in telephone consultations [5]

\begin{tabular}{|c|c|c|c|}
\hline No & Type of error & Common examples & How to prevent \\
\hline 1. & Information gathering & $\begin{array}{l}\text { 1.Inadequate drug and allergy history } \\
\text { 2.Lack of key questions }\end{array}$ & $\begin{array}{l}\text { 1.Open question } \\
\text { 2.Triage protocol } \\
\text { 3.Checklist }\end{array}$ \\
\hline 2. & Relationship building & $\begin{array}{l}\text { 1.Clinician anger and frustration with } \\
\text { time limitation and busyness } \\
\text { 2.patient anger over unmet expectations }\end{array}$ & $\begin{array}{l}\text { 1.Attentiveness to verbal and } \\
\text { non-verbal clues } \\
\text { 2.Clarify reason for call } \\
\text { 3.Supervion and feedback }\end{array}$ \\
\hline 3 & Decision making & $\begin{array}{l}\text { 1.Premature decision making or too } \\
\text { early closure in the consultation } \\
\text { 2.Absent diagnosis } \\
\text { 3.Wellness bias }\end{array}$ & $\begin{array}{l}\text { 1. Fram problems in terms of } \\
\text { disease and illness } \\
\text { 2.Involve patients in decision } \\
\text { making }\end{array}$ \\
\hline 4. & Explanation and planning & $\begin{array}{l}\text { 1.Unclear instructions and treatment } \\
\text { explanation }\end{array}$ & $\begin{array}{l}\text { 1.Smaller chunks of } \\
\text { information } \\
\begin{array}{l}\text { 2.Ask patient to repeat } \\
\text { information to r check } \\
\text { understanding }\end{array}\end{array}$ \\
\hline
\end{tabular}

\section{Suggested approach to a telephone consultation [2]}

- Answer the telephone promptly.

- State your name.

- Obtain caller's name and telephone number.

- Record date and time of call.

- Record person's name, gender and age.

- Speak directly with the person who has a problem.

- Take a detailed and structured history.

- Provide advice on treatment.
- Specific advice regarding follow-up and when to contact a doctor.

- Summaries the main points.

- Request caller to repeat advice given.

- Ask if the person has any outstanding questions or concerns.

- Let caller disconnect first.

\section{Medico legal concerns-}

- Telephone triage is an accepted form of remote assessment. The medico legal aspects of this 
work are the same as any other form of medical or nursing practice.

- There is a lack of published studies and case reports addressing this specific area.

- There is, however, a lack of specific guidance when it comes to telephone triage, but the usual best practice regarding accessing training, keeping yourself up-to-date and knowing when you have exceeded your professional boundaries remain.

- It is said that if a doctor provides consultation over e-mail or telephone, he can be liable, if there is any negligence while treating the patient.

- Legal pundits advise that doctors should avoid providing such consultations, unless they are already treating the patient and are aware of all aspects of the case. In respect of new cases, the doctor should not prescribe without seeing the patient physically.

- Don't ever repeat a prescription at all unless you have seen the patient physically.

- Third party calls are common in telephone triage and a particular risk, which can be managed by practice protocols and effective training.

- Other considerations are confidentiality and consent, especially with answer phones and documentation of calls.[8]

- Practices of telephonic consultation must provide adequate clinical supervision for professional accountability.

- Visual and audio-visual recordings can help with this task [9].

\section{Question of professional fees}

There is nothing wrong in charging for telephonic consultation, because-

- Traditionally, telephonic advice was given free of cost, but doctor has spent his time and utilized professional skill.

- $\quad$ There are also benefits to patients and third-party payers, because telephone care is cost-effective compared with traditional face-to-face encounters and it saved considerable time and efforts for the patient [10].

- Doctor's work component of telephone care shares all the characteristics of in-office care except for the hands-on physical examination.

- There is also increased liabilities, risks and practice expenses of telephone care as a justification for payment [11]

- May be more time consuming (to gather information) from doctors prospective.
- Although it become easy for patient to seek advise but technically requires more detailed knowledge and expertise from doctors part.

- In fact, many associations of doctors in India have pass resolutions that telephonic consultations should be charged depending on the routine and emergency calls.

\section{Reimbursement for Telephonic Consultations}

Despite strong patient demand for care provided telephonically and high levels of patient satisfaction, doctors tend to limit their use of email and the telephone for patient care due to reimbursement problems with such non face-to-face care.

\section{Conclusion}

A telephone consultation may provide safe, better quality and cost-effective healthcare to selected clinical conditions of patients. Although it is very easy to make mistakes on telephonic advice due misinterpretation of information provided from patient side or third party, absence of visual clues and lack of physical examination. Hence, the healthcare professionals should follow a proper structure of telephone consultation. It is undoubtedly a convenient way of providing healthcare advice to patients. Listening attentively, using a wellstructured approach and knowing common pitfalls of this form of consultation would not only decrease the chances of errors, improve patient satisfaction but could also improve cost effectiveness indirectly. As the medico legal part of the telephonic consultation is same as that for clinical practice in person, no obvious ways of getting professional fee for advice given, it seems to be a challenging job for the clinicians. More research guidelines, proper training, specific medico legal guidelines and issue of professional fee need to be addressed for doctors to use this kind of service in benefit of patients.

\section{References}

1. Hallam L. You've got a lot to answer for Mr Bell. A review of use of the telephone in primary care. Fam Pract. 1989;6:47-57.

2. Car J, Sheikh A; Telephone consultations. BMJ. 2003 May 3;326(7396):966-9

3. Foster J, Jessopp L. Telephone consultation course: An evaluation report. Londan: Department of general practice and primary care, King's College of School of Medicine and Dentistry. 1997. 
4. Car J, Freeman GK, Partridge MR, Sheikh A. Improving quality and safety of telephone based delivery of care: teaching telephone consultation skills. Qual Safety Health Care 2004;13: 2-3.

5. Males $\mathrm{T}$, Telephone consultations in primary care: a practical guide. RCGP 2007. ISBN: 978-0-85084-306-4

6. 'Your health, your care, your say' - public consultation report, Dept of Health, UK, Jan 2006, cited from: https:/www.gov.uk/government/uploads/system/uploads/ attachment_data/file/272238/6737.pdf on 27th April 2014.

7. Bunn F, Byrne G, Kendall S; The effects of telephone consultation and triage on healthcare use and patient satisfaction: a systematic review. Br J Gen Pract. 2005 Dec;55(521):956-61.

\section{Short Communication}

8. Sokol DK, Car J; Patient confidentiality and telephone consultations: time for a password. J Med Ethics. 2006 Dec;32(12):688-9.

9. Making and using visual and audio recordings of patients - guidance for doctors, General Medical Council, 2002.

10. Braithwaite SS, Unferth NO. Phone fees: a justification of physician charges. $\mathrm{J}$ Clin Ethics. 1993;4:219-24.

11. Metzl K. Telephone advice: to charge or not to charge, that is the question. Pediatrics. 1998;102:969.

\section{How to cite this article?}

Patel U, Gedam DS, Patel NP, Ratre BK. Telephonic Consultation: Modern Era of some Unsolved Questions. Int J Med Res Rev 2014;2(2): 172-175. doi:10.17511/ijmrr.2014.i02.21 